American Journal of Applied Sciences 10 (7): 724-733, 2013

ISSN: 1546-9239

(C) 2013 B. Pardamean et al., This open access article is distributed under a Creative Commons Attribution

(CC-BY) 3.0 license

doi:10.3844/ajassp.2013.724.733 Published Online 10 (7) 2013 (http://www.thescipub.com/ajas.toc)

\title{
Disease Management Information System
}

\author{
Bens Pardamean, Anindito, Anjela Djoeang and Nana Tobing \\ Department of Information Technology, \\ Faculty of Computer Science, Bina Nusantara University, Jakarta 11530, Indonesia
}

Received 2012-12-10, Revised 2013-04-06; Accepted 2013-07-02

\begin{abstract}
The study designed an information system model for Disease Management (DisMan) that met the specifications and needs of a consumer electronics manufacturer. The diseases monitored by this study were diabetes, hypertension and tuberculosis. Data were collected through interviews with the company's human resources department and occupational health provider. As for the model, literature and online research were conducted to collect health standards and information system standards on existing DisMan systems. These standards were then compared to the consumer electronics manufacturer's DisMan. The model was designed by utilizing class, use-case, as well as sequence diagrams. The result was an information system model with a registry system that can specifically support DisMan in a consumer electronics manufacturer. The registry system included a medical alert management system and a self-monitoring system. The latter system was supported by the former system, which can assist the DisMan's staff in monitoring the employees' scrupulousness regarding their health care plan as well as notifying the employees on lifestyle management.
\end{abstract}

Keywords: Information System Model, Disease Management, Self-Monitoring, Alert Management System, Medical Care Plan

\section{INTRODUCTION}

Employee productivity has a significant impact on a company's welfare. Therefore, employee issues are also a major concern to the company. One of the more important issues is that of employee health.

In handling employee health issues, companies often cooperate with healthcare service providers, who in turn offer services, such as disease prevention and treatment. These services are implemented as an effort to build employee awareness and interest regarding their health.

Disease management is the healthcare practice of treating patients, whom in this case are employees with chronic diseases that require high-cost treatments. These diseases also tend to have multiple treatment options. This is where disease management plays a crucial role since it entails treatment strategy for patients with chronic diseases; its ultimate role is the prevention of disease advancement.
On a related note, not every known disease has a cure. Nevertheless, a treatment is still required in order to prolong patient survival by minimizing the effect of the disease.

Chronic Care Model (CCM) proposed by (Wagner et al., 1996) is a form of disease management. $\mathrm{CCM}$ is used as a conceptual framework to handle patients with chronic diseases. CCM has the following components: self-management support, decision support, delivery system support, clinical information support, organizational of healthcare and resources and policies (Beich, 2006; Bodenheimer et al., 2002; Frei et al., 2010; Langford et al., 2007; Nutting et al., 2007; Strickland et al., 2010; Zai et al., 2008). CCM was developed in the United States and is currently implemented by the World Health Organization.

In this study, the researchers discuss an information system model as a support for disease management. Employees with chronic diseases, specifically

Corresponding Author: Bens Pardamean, Department of Information Technology, Faculty of Computer Science, Bina Nusantara University, Jakarta 11530, Indonesia 
hypertension, diabetes and tuberculosis, can be medically monitored to prevent health deterioration caused by the disease. Various studies have shown that if people were to have access to more detailed medical information, it would be more likely for them to develop an interest in their health, leading to a more pro-active involvement in the decision-making process concerning their health (Mocean et al., 2009).

Various studies have been conducted to substantiate the essential roles that information technology carries within the health industry. One such study is the implementation of information technology to support disease management.

Patients with chronic illnesses need to have their health monitored to maintain a stable condition. Supervising patient conditions could lead to improvements in post-treatment patient condition as well as potentially reduction in the cost of treatment. With assistance from the medical staff (such as nurses), patients can learn to self-monitor their health.

Patients are equipped with the tools needed to obtain current state of health measurements (Alaoui et al., 1998) and to check the tools connected to the transmitter. Data measurement results are sent to the servers managed by the medical staff. Patients can view and ensure that the reading results have been received by the server through a customized personal web page (Leijdekkers et al., 2007; Pomazan et al., 2009; Shahriyar et al., 2009).

Mobile phones can also be used to enter and record the results of the measurements using voice commands, while the medical staff monitors daily activities of the patient through the web. The staff provides a response through a system that provides alerts and readings in the form of tabulated data and graphs on the web (Harper et al., 2008).

Monitoring can also be done by installing an equipment in the patient's home that serves as a proactive agent in which the monitor takes periodic data samples from patients at a predetermined time. The monitor sends the data and reports unusual events to an expert system via the Internet (Zhang et al., 2009).

The goal in Disease management (DisMan) is to maintain employee health. This is implemented by raising awareness for their own health conditions. The role of the DisMan staff is to formulate a medical care plan for each employee. The medical care plan then assists patients to achieve a predetermined target. In the current process, DisMan was not conducted optimally since the DisMan staff could not evaluate the effectiveness of the assigned medical care plans.
The lack of an information system capable of monitoring employee health has conceived the following problems for the DisMan staff: (1) How to monitor a medical care plan for a patient and help them achieve the predetermined targets and (2) How to raise patient awareness and adherence with the given recommendations.

\section{MATERIALS AND METHODS}

Literature review was performed to obtain a guideline for the system design. This was performed by comparing healthcare standards for diabetes, hypertension and tuberculosis from various sources; the current health care standards were set by the consumer electronics manufacturer. The result of this study was an information system model that supported the care of diabetes, hypertension and tuberculosis at a consumer electronics manufacturer. The routine examinations included the monitoring of employee meal intakes, regular training, health program participation and weekly consultation with a physician.

This monitoring was supported by the help of alerts that helped in ensuring a patient to: (Aiko, 1995) record measurements of current health conditions; (Alaoui et al., 1998) self-administer medication with the recommended dosage on time and (Beich, 2006) follow the exercise program designed by DisMan staff. Due to the huge amount of data, it may be difficult for physicians to decide on the best procedure that could provide the best possible care (Santos et al., 2009).

The availability of this system was expected to provide better information for DisMan staff and their patients. The system aided the DisMan Staff in gathering patient information, which were collected and analyzed for possible diagnoses and treatments for a particular patient (Santos et al., 2009; Matamoros et al., 2005).

\section{RESULTS}

Figure 1 depicts the DisMan system that is currently implemented in a consumer electronics manufacturer. Employees that are members of a DisMan plan have the obligation to follow a medical care plan that was previously mutually agreed upon with the DisMan staff. Medical care plan includes various targets that must be attained by the employee, such as composition and quantity of nutrition that must be consumed, physical activity duration that must be accomplished following a prescheduled DisMan program to raise awareness of their illnesses and available treatments or medication and the next consultation schedule with a physician. 


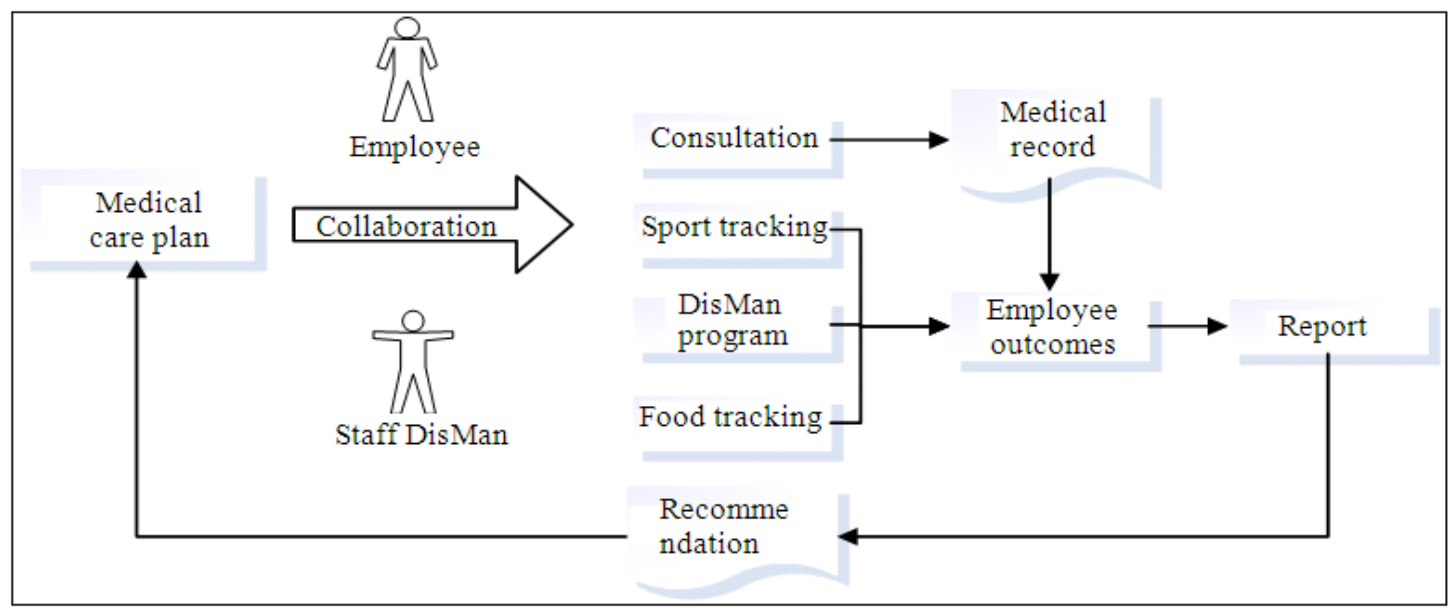

Fig. 1. Current DisMan system

Currently, employees have a weekly physician's consultation provided by the health care provider. Blood glucose and blood pressure measurements are conducted during this clinical visit. During consultation, the medical staff, mainly nurses, educates the patient on healthy lifestyle choices. The medical staff consists of work safety physicians, assistant physicians (the person in charge), nurses, nutritionists and pharmacists. Every patient is given a medical care plan with targets determined by the work safety physician along with the DisMan staff. This medical care plan is later incorporated into a monthly health report for each patient. Employees are also permitted to obtain a second opinion from their own physicians, provided that they report additional medications along with their dosages. The DisMan program at the consumer electronics manufacturer currently focuses on the monitoring of sodium intake, with the target of lowering the daily value.

DisMan staff regularly checks an employee's conditions and assesses whether or not the medical care plan targets are on track. During consultation, a DisMan staff investigates the reason for an employee's deviation from the medical care plan. After obtaining the information and considering examination results during consultation, the DisMan staff files a report, which in turn leads to modifications for the employee's subsequent medical care plan.

Current employee health care plan is considered suboptimal since the medical staff has not been able to monitor the most up-to-date developments. The latest condition of the patient is only obtained during a visit to the clinic (at the end of the Medical Care Plan).
Consultation is also time consuming since the DisMan staff must verify: All health program(s) undergone by patients, the last medical care plan and patient's work environment. Thus, all the information needed for care is actually available but they must be searched manually.

Patients also do not have full motivation to keep their lifestyle and health patterns. This is observed from the frequent non-adherence of patients towards recommendations from the DisMan staff, such as lack of participation in exercise programs. Patients also tend to get their health condition and other information required by the DisMan staff.

Based on previous chronic disease management systems, important criteria to that this study considered were:

- Importance of information in determining further action to be taken (Pomazan et al., 2009; Dai et al., 2008; Tong, 2006; Paganelli and Giuli, 2007; LeRouge et al., 2010; Tura et al., 2005)

- Distance as an obstacle in obtaining patient data (Dai et al., 2008; Tong, 2006; Paganelli and Giuli, 2007; LeRouge et al., 2010)

- Automatic measurement in reducing data inaccuracies (Tong, 2006; Paganelli and Giuli, 2007; LeRouge et al., 2010; Tura et al., 2005)

- Expert systems in assisting physicians during patient diagnosis (Pomazan et al., 2009; Dai et al., 2008)

Figure 2 describes the proposed design of the DisMan system under this study. Explanation of the figure is as follows: 


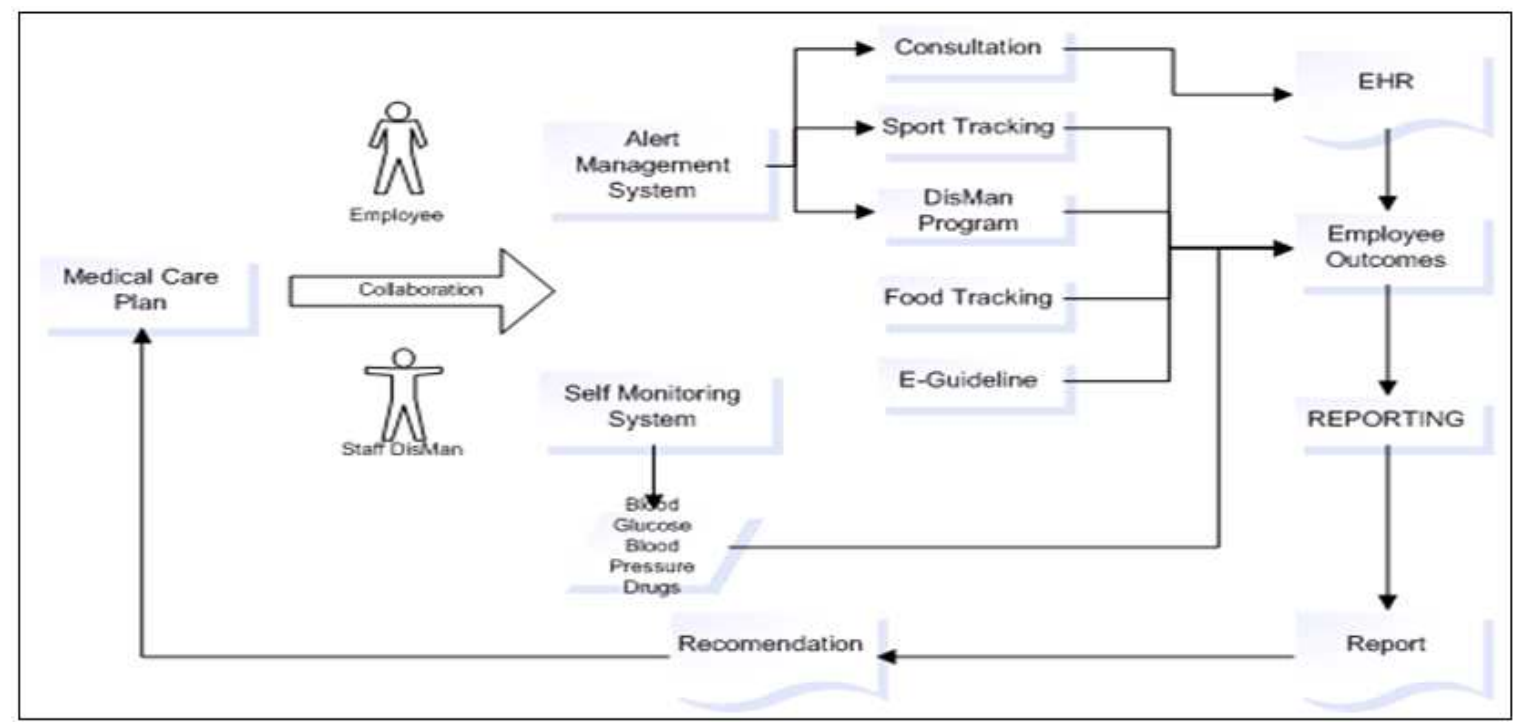

Fig. 2. Proposed DisMan system workflow

- DisMan participants are employees diagnosed with hypertension, diabetes and/or tuberculosis

- DisMan staff creates a medical care plan to be followed by patients for a certain period of time before the next visit. The medical care plan contains targets that must be achieved by the patient (blood pressure, blood glucose, medication, nutrition and exercise)

- DisMan staff creates notifications in accordance with the requirements of each patient

- System sends notifications to patient to undertake measurements of blood glucose and blood pressure. Notifications are also sent for medication time, nutrition, exercise activity reminders, DisMan programs attendance and visitation time

- Blood glucose, blood pressure medication, exercise activity and DisMan program attendance data are kept in a database

DisMan staff created the latest medical care plan based on information provided by the system. Data from the system was accessible as needed. The created medical care plan was always compared with previous targets from the previous medical care plan.

Electronic Health Records was an important component in understanding patient conditions. During patient evaluation, information regarding the clarification of the disease, detection of complications, review of previous treatments and glycemic control for diabetic patients should be indicated. This led to the formulation of subsequent medical care plan by providing a basis for further treatment.

IT Role in supporting Chronic Care Model focused on Clinical Information Support namely Registry System that consisted of Electronic Health Record, Alert Management System and Self Monitoring System. Alert Management System and Self Monitoring System aimed to support patient self-management. Patient education as a treatment step could be supported by health appointment schedules and electronic guidelines.

The designed system was a web-based system that presented information in real-time access, allowing for a concurrent monitoring of employee health conditions. The information provided was taken into consideration while designing further medical care plan to maintain employee health. The system could be accessed by the DisMan staff, DisMan program participants and employees who have been trained to run the DisMan system. This information system was operated with a PC, server and smart phones. Objects in the system design were Employee, Department, Medical Record, Drugs, Food Tracking, Glucose, Blood Pressure, Report, DisMan Membership, Consultation, Physician, Care Plan, Supervisor, Alert, Urgency Level, Program, Guideline and Sport Attendance. Data access Application Programming Interface (API) were designed for accessing and utilizing the database. The API provided user interface for data access requests (Jicai et al., 2011). 
The designed system was intended for supplying information to the DisMan staff. The information included data consisting of blood glucose, blood pressure, medication and exercise attendance. Usage of this information aimed to modify current medical care plan at any time if a deviation from the target were to occur.

For patients with diabetes, the considered factors were the monitoring of glycemic levels (blood glucose monitoring or A1c measurement), medical nutrition therapy and physical activity.

For patients with hypertension, the considered factors were stable blood pressure, lifestyle modification and regular administration of medication.

For patients with tuberculosis, the considered factors were administration of each medication type at each of its correct dosage since patients with tuberculosis have a chance of full recovery.

The DisMan staff could be divided into several agents, leading to an optimal primary care (Daknou et al., 2008), namely:

- Home Agent is assigned with creating patient records and conducting observations during treatment

- Problem Identifier Agent is responsible for determining disease severity and subsequently, a treatment plan based on the proper protocols

- Scheduler Agent is responsible for determining the duties and responsibilities of each agent in the medical staff after obtaining a summary report of patient condition

- Monitor Agent is assigned to patients that require more intensive treatments. Monitor Agent works closely with the Problem Identifier Agent to determine the latest patient condition

Previous study (Paganelli and Giuli, 2007) describes a home-based service application framework for monitoring and handling chronically ill patients by providing medical status information and an alert mechanism. Most chronic diseases need special care from the nursing staff in order to notify patients of the time to administer the correct medication (Chun-Liang, 2007).

Alert Management System consists of two parts, namely alert creation module (Incoming Alert Monitor) and execution module (Outgoing Alert Monitor), in which the execution module is further divided into three sub-modules namely: role matching module, alert monitor module and priority urgency module (Chiu et al., 2004). The designed Alert Management System is shown in Fig. 3.

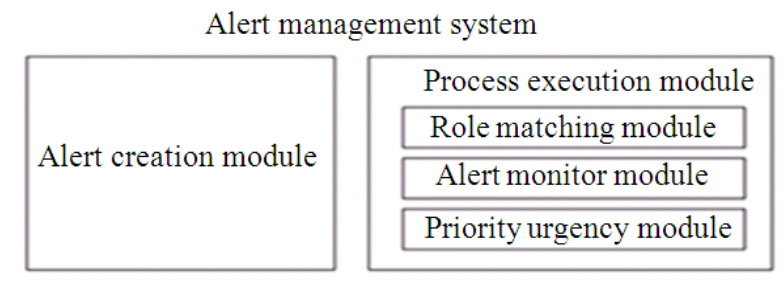

Fig. 3. Division of the alert management system

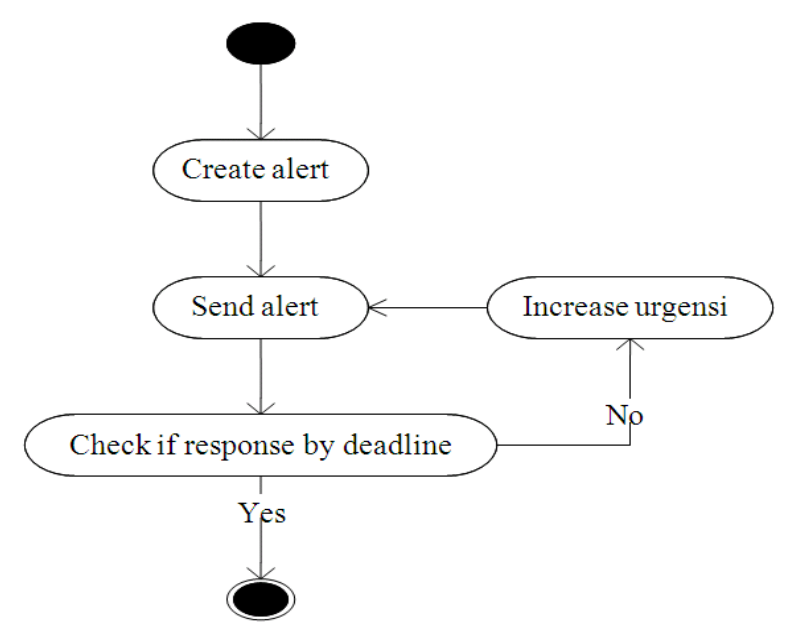

Fig. 4. Alert execution flowchart

Table 1. Alert urgency level and actions

\begin{tabular}{ll}
\hline Urgency & Action \\
\hline Normal & Email and send SMS to patient \\
Urgent & Send SMS to patient \\
Critical & Send SMS to DisMan staff \\
\hline
\end{tabular}

The process execution module will provide a response to incoming alerts as shown in Fig. 4. Incoming alerts shall be monitored and put into a queue and will be given appropriate services. Any pending alerts are submitted in accordance with the web service request. Priority urgency module defines the rules to be executed if the alert receives no response within a certain time limit (deadline).

Alert status information will be updated after the alert has received a response. If the alert in a given time does not obtain a response (categorized as expired) then the module checks the urgency strategy table then executes corresponding action and updates the corresponding status information. In the Alert Management System urgency level is classified into three levels as depicted in Table $\mathbf{1 .}$

Table 1 indicates that urgency function $U(t)$ is defined as follows: 


$$
\mathrm{U}(\mathrm{t})=\left\{\begin{array}{l}
\text { Normal, } \mathrm{t}=\mathrm{T} \\
\text { Urgent, } \mathrm{T}<\mathrm{t}<\mathrm{T}+\mathrm{dt} 1 \\
\text { Critical, } \mathrm{T}+\mathrm{dt} 1<\mathrm{t}<\mathrm{T}+\mathrm{dt} 1+\mathrm{dt} 2
\end{array}\right.
$$

Where:

$$
\begin{aligned}
\mathrm{T} & =\text { Normal deadline (default) } \\
\mathrm{dt} 1 & =\text { Urgent deadline } \\
\mathrm{dt} 2 & =\text { Critical deadline }
\end{aligned}
$$

If patient fails to respond on a $\mathrm{T}$ normal deadline, the urgency level is raised from normal to urgent, leading to the resending of the alert. Should there be no response after the urgent deadline, the urgency level will be raised to critical and another alert is resent.

Based on the urgency level, there are five scenarios:

- System sends alert to patients to check their blood glucose levels (diabetes)

- Systems sends alert to patients to check their blood pressures (hypertension)

- System sends alert to patients to attend a medical check-up at the clinic (diabetes, hypertension, Tuberculosis (TBC))

- System sends alert to patients to attend DisMan sport or education activity (diabetes, hypertension, TBC)

- System sends alert to patients to take medication (diabetes, hypertension, but more geared towards TBC patients)

When alert urgency reaches a critical level, a text message is sent to the DisMan staff, who would then contact the corresponding patient. This may be done via various communication technologies, such as a wideband mobile radio channel (Takizawa, 2007a; 2007b).

Self-Monitoring of Blood Glucose (SMBG) aids in controlling diabetes by providing information on blood glucose development for diabetic patients while SelfMonitoring of Blood Pressure (SMBP) provides blood pressure information for patients with hypertension.

Data are obtained according to a determined schedule. For SMBG or SMBP, when the data is not aligned with the predetermined medical care plan, the DisMan staff may directly consider a change in medical care plan regarding meal consuming, sport activity and medication intake.

With the presence of daily blood glucose and blood pressure information, it is anticipated that patient awareness is raised, leading to motivation to maintain dietary patterns and physical activities.
Patients with diabetes check blood glucose levels with a glucose meter (Fig. 5) that sends the data to the patient mobile phone via Bluetooth (Fig. 6). Blood glucose level is then sent by the mobile phone to the health care provider database. The Bluetooth profile used is Serial Port Profile (SPP), which controls data access by defining necessary procedures for configuring connections between Bluetooth devices using RFCOMM. Bluetooth technology is chosen due to its relatively low power consumption (Tafa and Stojanovic, 2006).

User interface of the blood glucose meter shows blood glucose level, time taken, qualitative description (high or low) and description of pre- and post-meal blood glucose levels. Afterwards, patients are asked to download data to their mobile phones just by the press of a button. Once all data is downloaded, the mobile phone user interface will show all the collected data in an organized display. Then with another press of the button, the patient is asked to send these data to the DisMan database. Patient are able to see their blood glucose level for a certain time period by inputting start and end dates on their mobile phones.

The gray area in Fig. 7 encompasses targets to be achieved by the patient for blood glucose level during a time period of the medical care plan compiled by the DisMan staff. Result comparisons between different measurements and the determined targets in a medical care plan period are shown with the anticipation of developing patient awareness. Alert procedures for patients with hypertension have the same procedures with those for patients with diabetes in terms of measurements and medication administration. Continuous monitoring of physiological parameters, such blood pressure and blood glucose levels, could remarkably reduce the risk of serious complications in cases of patients with chronic diseases (Tafa and Stojanovic, 2006).

A result of this study was a registry system model that functioned as an information system that supported the implementation of disease management at a consumer electronics manufacturer. The system's capability included:

- Daily monitoring of patient conditions. DisMan staff was able to determine when the patient conditions deviated from the planned target then take immediate actions. The latest patient condition monitoring results were then set as a guideline for restructuring the current medical care plan 


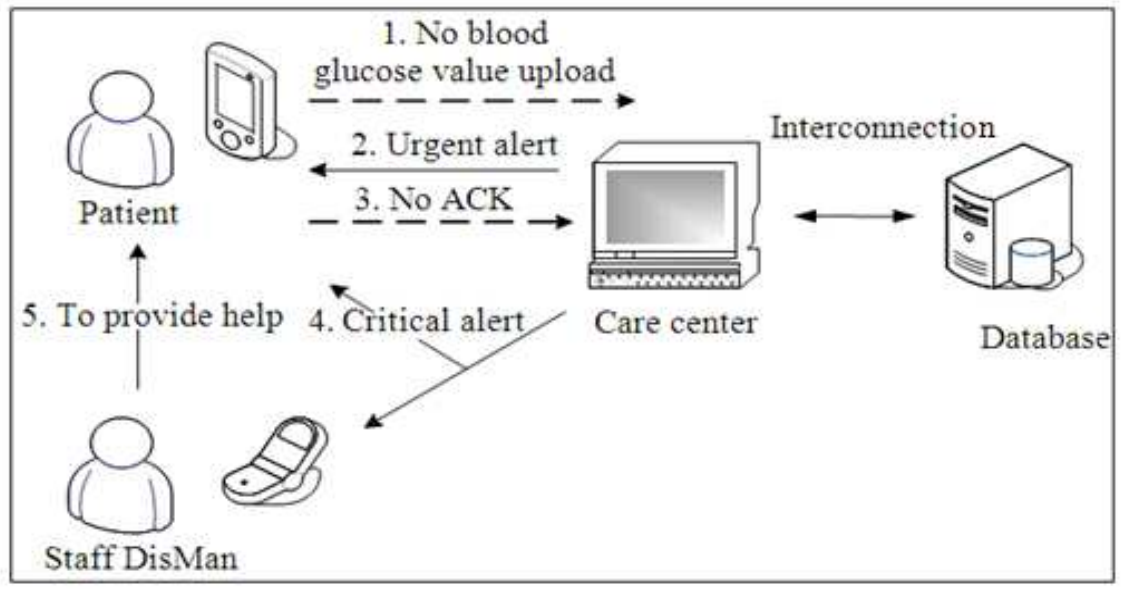

Fig. 5. Alerting procedure for patients to have blood glucose level check

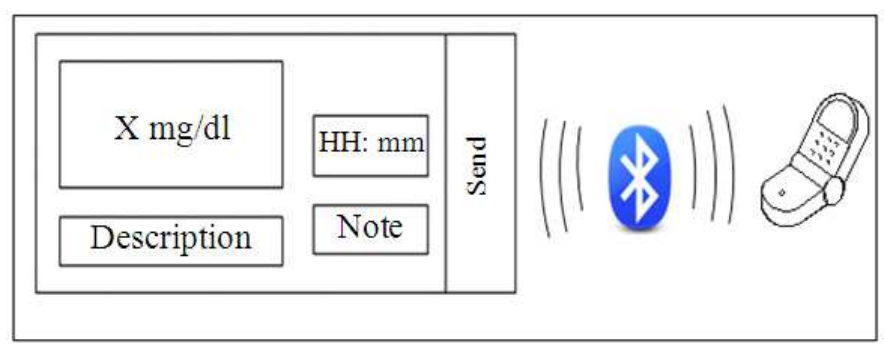

Fig. 6. Blood glucose monitor connected to patient mobile phone via Bluetooth

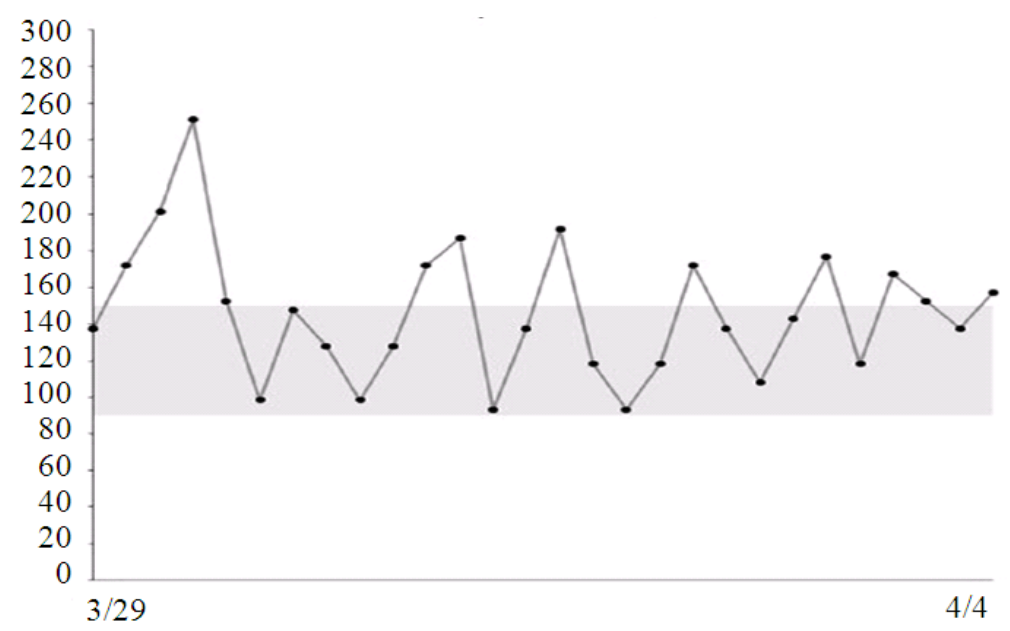

Fig. 7. Patient User Interface to display blood glucose measurement in a certain period

- Patient awareness raising leading to adherence with recommendations from the DisMan staff. Patients were reminded by the Alert Management System to perform measurements according to the predetermined schedule
Patients could also view the measurement results from the Self-Monitoring System and compare them with their targets. Information obtained by the registry system was utilized for analysis by the DisMan staff to help determine subsequent medical care plans. This 
system assisted in providing data as information for DisMan staff in monitoring patient conditions.

\section{DISCUSSION}

An electronics manufacturer implemented a DisMan program without the usage of information technology. The most common DisMan programs were exercise programs and promotion of low sodium meals in the factory cafeteria for employees with hypertension. DisMan staff could determine which employees were on a DisMan program during time of consultation. For instance, if the condition of a patient with hypertension were to deteriorate relative to previous conditions, reports on the low sodium diet counter and exercise attendance could be verified.

The designed system allowed both the DisMan staff and the patients to monitor the latter's current condition to obtain the required information. Self-monitoring system with an alert management system could help the DisMan staff in evaluating patient adherence to the health care plan that had been mutually determined. The system also indirectly helped in raising awareness for the patients to control their lifestyles. Patient selfmonitoring data results would be information for DisMan staff and later utilized to design subsequent treatment plans. Thus, the Disease Management system could be optimized because of the availability of patient monitoring information, which could also be cross-checked with targeted goals.

The designed registry system was formulated for patients with diabetes, hypertension and tuberculosis. The system allowed for the availability of a better data management system with alerting features that would remind patients to perform self-monitoring according to a predetermined schedule. Patients also no longer had to wait until the next scheduled consultation in order to ascertain their latest conditions. In the process, patient awareness with regard to health status is increased since patients would be more concerned about their health due to the routine measurements.

Patients no longer had to visit special facilities to take measurement of blood glucose level, blood pressure, or access their own health information. All of this could be done anywhere and anytime through the use of provided measuring devices combined with mobile phones. To reduce errors, data from gauges were sent via Bluetooth to patients' mobile phones before the forwarding to the DisMan staff's servers.

\section{CONCLUSION}

The registry system is web-based, which enabled patients to access their own data. The DisMan staff was able to view consultation schedules, review HER, check patient status, check the diet tracking, check sports attendance, inspect DisMan participation program, view reports, create reports, manage the treatment of patients, schedule consultations, providing e-guidelines and schedule alerts. This registry system is not yet equipped with an expert system for diagnosis or a subsequent health care plan designer. Health monitoring of chronic disease patients is instrumental in the implementation of DisMan. The DisMan program is said to be successful when once it is able to assist patients in achieving a stable or better condition with regards to their chronic disease(s).

\section{REFERENCES}

Aiko, H., 1995. Domestic Violence. In: Japanese Women: New Feminist Perspectives on the Past, Present and Future, Fujimura-Fanselow, K. and A. Kameda, (Eds.), Feminist Press at CUNY, ISBN-10: 1558610944, pp: 265-267.

Alaoui, A., S. Clement, N. Khanafer, J. Collman and B. Levine et al., 1998. Diabetes home monitoring project. Proceedings of the Symposium on Pacific Medical Technology, Aug. 17-20, IEEE Xplore Press, Honolulu, Hawai, pp: 258-261. DOI: 10.1109/PACMED.1998.769917

Beich, J., 2006. The role of disease management in payfor-performance programs for improving the care of chronically ill patients. Med. Care Res. Rev., 63: 96S-116S. PMID: 16688926

Bodenheimer, T., E.H. Wagner and K. Grumbach, 2002. Improving primary care for patients with chronic illness. J. Am. Med. Assoc., 288: 1775-1779. PMID: 12365965

Chiu, D.K.W., B.W.C. Kwok, R.L.S. Wong, S.C. Cheung and E. Kafeza et al., 2004. Alerts for healthcare process and data integration. Proceedings of the 37th Hawaii international Conference on System Sciences, Jan. 5-8, IEEE Xplore Press, Big Island, pp: 60146-60146. DOI: 10.1109/HICSS.2004.1265371

Chun-Liang, H.S.U., 2007. Intelligent reminder system of having medicine for chronic patients. Proceedings of the 11th WSEAS International Conference on Communications, (ICC' 07), Crete Island, Greece, pp: 202-207. 
Dai, S., Y. Gui and X. Zhu, 2008. Design of the intelligent real-time hypertensive diagnosis expert system based on web. Proceeding of the International Symposiums on Information Processing, May. 23-25, IEEE Xplore Press, Moscow, pp: 252-256. DOI: 10.1109/ISIP.2008.136

Daknou, A., H. Zgaya, S. Hammadi and H. Hubert, 2008. Agent based optimization and management of healthcare processes at the emergency department. Int. J. Math. Comput. Simulat., 2: 285-294.

Frei, A., C.C. Moshinsky, H. Schläpfer, B. Birnbaum and U. Held et al., 2010. The chronic care for diabetes study (CARAT): A cluster randomized controlled trial. Cardiovascular Diabetol., 9: 1-11. DOI: $10.1186 / 1475-2840-9-23$

Harper, R., P. Nicholl, M. McTear and J. Wallace, 2008. Automated phone capture of diabetes patients readings with consultant monitoring via the web. Proceedings of the 15th Annual IEEE International Conference and Workshop on the Engineering of Computer Based Systems, Mar. 31-Apr. 4, IEEE Xplore Press, Belfast, pp: 219-226. DOI: 10.1109/ECBS.2008.31

Jicai, W., R. Tingguang and Z. Jing, 2011. The methodology, meta-synthetic integrated environment and data accessing service of stratagem research. Int. J. Math. Comput. Simulat., 5: 520-527.

Langford, A.T., D.R. Sawyer, S. Gioimo, C.A. Brownson and M.L. O'Toole et al., 2007. Patientcentered goal setting as a tool to improve diabetes self-management. Diabetes Educator, 33: 139S144S. DOI: 10.1177/0145721707304475

Leijdekkers, P., V. Gay and E. Lawrence, 2007. Smart homecare system for health tele-monitoring. Proceedings of the 1st International Conference on the Digital Society, Jan. 2-6, IEEE Xplore Press, Guadeloupe, pp: 3-13. DOI: 10.1109/ICDS.2007.37

LeRouge, C., M. Gaynor, C.C. Li and J. Ma, 2010. A multi-level technical infrastructure for diabetes chronic care management in china. Proceedings of the 43rd Hawaii International Conference on System Sciences, Jan. 5-8, IEEE Xplore Press, Honolulu, HI, pp: 1-10. DOI: 10.1109/HICSS.2010.21

Matamoros, A., A. Torrealba, F. Rivas S. González and R. Sánchez et al., 2005. Expert system for the preeclampsia prevention program. Proceedings of the 4th WSEAS International Conference on Computational Intelligence, Man-machine Systems and Cybernetics, (MSC' 05), Miami, USA., pp: 146-149.
Mocean, F., L. Lotrean, L. Filip, C. Borzan and M. Baritz, 2009. Using modern information technology in health promotion through various forms of health education at community. Proceedings of the 11th WSEAS International Conference on Mathematical Methods and Computational Techniques in Electrical Engineering, (TEE' 09), pp: 458-462.

Nutting, P.A., W.P. Dickinson, L.M. Dickinson, C.C. Nelson and D.K. King et al., 2007. Use of chronic care model elements is associated with higherquality care for diabetes. Ann. Fam. Med., 5: 14-20. PMID: 17261860

Paganelli, F. and D. Giuli, 2007. An ontology-based context model for home health monitoring and alerting in chronic patient care networks. Proceedings of the 21 st International Conference on Advanced Information Networking and Applications Workshops, May 21-23, IEEE Xplore Press, Niagara Falls, Ont, pp: 838-845. DOI: 10.1109/AINAW.2007.90

Pomazan, V.M., L.C. Petcu, S.R. Sintea and R. Ciorap, 2009. Active data transportation and processing for chronic diseases remote monitoring. Proceedings of the International Conference on Signal Processing Systems, May 15-17, IEEE Xplore Press, Singapore, pp: 853-857. DOI: 10.1109/ICSPS.2009.120

Santos, M.F., F. Portela, M. Vilas-Boas, J. Machado and A. Abelha et al., 2009. Nursing information architecture for situated decision support in intensive care units. Proceedings of the 9th WSEAS International Conference on Applied Informatics and Communications (AIC '09), Moscow, Russia, pp: 491-496.

Shahriyar, R., B.M.D. Faizul, K. Gourab, A.S. Iqbalet and A.M. Mostofa, 2009. Intelligent Mobile Health Monitoring System (IMHMS). Int. J. Control Automat., 2: 13-27. DOI: 10.1007/978-3-64211745-9_2

Strickland, P.A., S.V. Hudson, A. Piasecki, K. Hahn and D. Cohen et al., 2010. Features of the Chronic Care Model (CCM) associated with behavioral counseling and diabetes care in community primary care. J. Am. Board Fam. Med., 23: 295-305. DOI: 10.3122/jabfm.2010.03.090141

Tafa, Z. and R. Stojanovic, 2006. Bluetooth-based approach to monitoring biomedical signals. Proceedings of the 5th WSEAS International Conference on Telecommunications and Informatics, (TI’ 06), Istanbul, Turkey, pp: 415-420. 
Takizawa, Y., S. Yatono and A. Fukasawa, 2007a. Advanced synchronization scheme for wideband mobile communications. Int. J. Math. Mod. Meth. Applied Sci., 1: 315-319.

Takizawa, Y., S. Yatono and A. Fukasawa, 2007b. High performance digital signal processing system for wideband mobile communications. Int. J Math. Comput. Simulat., 1: 146-149.

Tong, D.A., 2006. Development of a networked blood pressure monitoring system. Proceedings of the 1st Transdisciplinary Conference on Distributed Diagnosis and Home Healthcare, Apr. 2-4, IEEE Xplore Press, Arlington, VA., pp: 129-132. DOI: 10.1109/DDHH.2006.1624814

Tura, A., L. Quareni, D. Longo, C. Condoluci, A.V. Rijn and G. Albertini et al., 2005. Wireless home monitoring and health care activity management through the Internet in patients with chronic diseases. Med. Inform. Internet Med., 30: 241-253. PMID: 16531352
Wagner, E.H., B.T. Austin and M.V. Korff, 1996. Organizing care for patients with chronic illness. Milbank Q., 74: 511-544. PMID: 8941260

Zai, A.H., R.W. Grant, G. Estey, W.T. Lester and C.T. Andrews et al., 2008. Lessons from implementing a combined workflow-informatics system for diabetes management. J. Am. Med. Inform. Assoc., 15: 524533. DOI: $10.1197 /$ jamia.M2598

Zhang, Y., M. Lee and T.M. Gatton, 2009. Agent-based web healthcare systems for real-time chronic disease. Proceedings of the World Conference on Services-I, Jul. 6-10, IEEE Xplore Press, Los Angeles, CA., pp: 14-21. DOI: 10.1109/SERVICESI.2009.104 